\title{
Pattern Recognition in Games
}

\author{
Dhruvil Parmar, Mit Suthar, Dhaval Bhoi
}

\begin{abstract}
In this paper, we discuss how pattern recognition plays a vital role in games [3]. Pattern recognition is used to gather necessary details related to the gaming world and that will provide us input to the decision-making system, which produces actions for the game world [1]. Before pattern recognition how the decision is making and implementing it. We also define emotion-based recognition in affect-aware games [11]. Then we discuss the usefulness of pattern recognition in games, how and where it can be implemented and what is expected from it and how it can affect relations between the human player and synthetic player [1].
\end{abstract}

Keywords: Computer generated player(Bots), Decision-making, Operational, Strategical, Tactical.

G ame developers have an idea about the gaming world so that it is the most eligible area to apply pattern recognition. Current modern computer games have high graphics and sound but also, they have a synthetic player or we can say computer-generated player which challenges the human player. When a human player plays so many times with bots (Computer-controlled player), the human player finds a pattern in bots and it can easily exploit their weaknesses. Because of this problem the gaming industry is moving towards AI-based bots. In this context, pattern recognition is very useful to take out relevant details from the gaming world and make the concepts. These concepts are based on high-level reasoning and decision making which is associated with artificial intelligence problems.

Pattern recognition is not a whole AI system but it is a part of it. It includes several classification techniques. When the human player interacts with the synthetic player, the pattern recognition machine will extract relevant information of human player and this information will be helpful in the decision-making system which is basically a part of the AI system.

The main motive to do pattern recognition is to discuss where and how it can be applied in games. We will discuss how pattern recognition plays a role in the relation between the human player and synthetic player. We try to understand the importance of pattern recognition in games.

\section{RELATED WORK}

Pattern recognition is widely useful in computer games for engaged players. Nowadays, every game developing

Revised Manuscript Received on May 07, 2020.

Dhruvil Parmar, Computer Engineering, CSPIT CHARUSAT, Anand, India. Email: 17ce067@charusat.edu.in

Mit Suthar, Computer Engineering, CSPIT CHARUSAT, Anand, India. Email: 17ce123@charusat.edu.in

Dhaval Bhoi, Computer Engineering, CSPIT CHARUSAT, Anand, India. Email: dhavalbhoi.ce@ charusat.ac.in

\section{INTRODUCTION}

companies are trying to implement Artificial Intelligence to improve the game experience for players. In the present era, affect-aware games are being developed by companies that run based on the player's emotions. In this type of game, it uses affect state models that identify the player's emotion and then based on the emotional decision-making system changes a game storyline [11]. Now based on emotions, the data-pattern machine is trying to understand the emotion of the real player and in further upcoming moves, it provides the moves which are related to the previously generated results.

Previously for the AI portion of games, they are going to recognize only the object around the main element and based on the movement of them and their position they are trying to make a decision [8]. But in this, we define not only moves that are done based on the game elements but also done after recognizing each and every situation's decision which are taken by a real player and try to correlate them to perform the effective move for a particular situation.

\section{FUNDAMENTALS OF PATTERN RECOGNITION}

Pattern recognition works as a portion of the whole AI system. The AI system has mainly two components. The first one is pattern recognition which extracts information data sources. Here, the data source can be the game world. That extracted information is provided as an input to the decision-making component. There are some AI applications that require a higher level of reasoning which is also based on information that is extracted by pattern recognition.

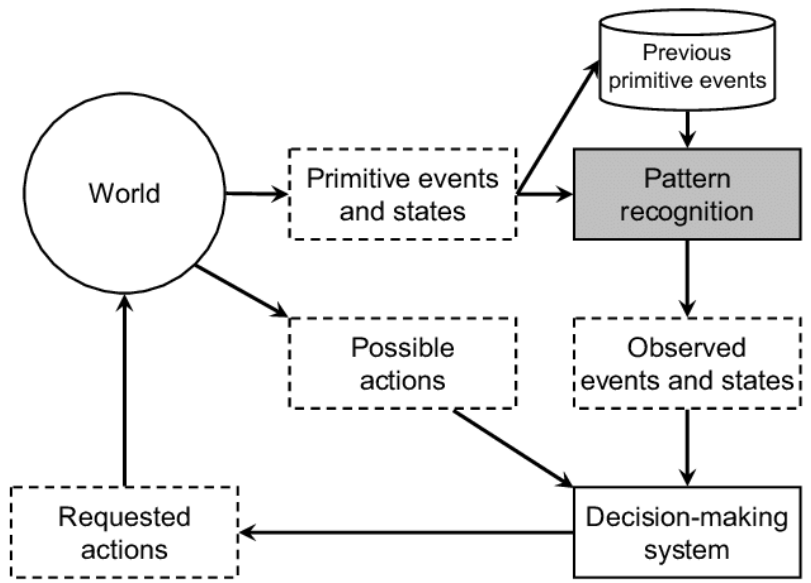

Fig. 1. how pattern recognition is connected with the virtual world and decision-making [1]

Fig. 1 represents the relation between world, pattern recognition and decision making. Based on human player interaction with the game world it will maintain primitive events and states. 


\section{Pattern Recognition in Games}

From current primitive events, states and previous primitive events, pattern recognition generates observations that are forwarded to the decision-making system. There are possible actions performed in the world which directly leads to decision-making system where it provides responses based on observed events and states by pattern recognition.

The actual phenomena of the world that we want to identify are known as a pattern. Pattern recognition approaches were classified into statistical, structural and neural [7]. A statistical approach, in which results can be drawn out from established concepts in statistical decision theory in order to discriminate among data based upon quantitative features of the data from different groups. It includes a supervised and unsupervised learning process. In the supervised learning process, the designer already provides current identification for all possible inputs. It means whenever any input which matches with predefined identification then predefined actions will perform. In the unsupervised learning process, the main goal is to create classes with similar inputs performed by clustering methods. A structural approach is in which results can be produced from interconnections and interrelationships of the feature. The neural pattern recognition approach uses biological concepts into the machine to identify a pattern.

\section{PATTERN RECOGNITION USE IN GAMES}

Pattern recognition in-game can be analyzed from several views. In this following, we define how pattern recognition achieves a distinct level of culpable. After that, we characterize the duty of pattern recognition in a connection between a computer player and a real player. We discuss the open game world and story generation.

\section{A. Levels of Decision-Making Problems}

As per decision-making problem categories, there are three categories: strategical, tactical and operational.

On the Strategical decision level, pattern recognition requires a large amount of data and it requires a long period of time to work. It contains high-quality recognition methods. The reason for the high amount of data set is it can be gathered based on environment, assets and the ongoing activity in the game world. Clearly, in this decision level to get appropriate precision we need a large amount of data set but the amount of data required to generate a result is can't be able to handle normally. This level is implemented offline or in the background. The main reason to focus on the valuable result is the rate of failure is high at strategical level.

The tactical decision level is an intermediary level between the Strategical decision level and Operational level. The tactical decision level is one step lower than the Strategical level, that's why it requires less time to generate frequently compare to the strategical level. Same as the quality of the result can't be as high as the Strategical level. If the strategical level describes what should be done, then at the tactical level the result describes how to perform the result of the strategical level.

The operational decision level is closely associated with the properties of the gaming world or assets of the virtual world and that's why the pattern recognition techniques must be short-term and real-time. Because this decision is made for less period of time and less accurate pattern recognition doesn't necessarily lead towards the non-recoverable problem. For instance, if the chosen track of result leads towards a dead-end, then we can still reroute that tack until we get the desired output.

\section{B. Enemy Player or Team Player or Moderate Player}

In every game, we think that a computer as an enemy or as a team player. For example, a human player's interface could be implemented by a virtual player that can be able to report regarding an enemy's moments and also suggests the counter defense or counter moment in a real-time strategy game. In the above example, pattern recognition analyzes the human's perspective and generate the result and will be giving to the real player instead of passing to the decision-making mechanism.

Another duty of a computer player is to act as a moderate player. For example, in a cricket game or racing game, a commentator is a player who defines highlight the event and provides background information about the event. For example, RoboCupSoccer demonstrates AI simulated soccer league [6].

In Addition to artificial commentators, the computer acts as a self-determining camera director. For this camera, the director application requires a specific pattern recognition method. Suppose if any racing happens in real-time it requires a large number of cameras which having different angles based on the right place at which it placed and at a particular time which captured clip from which camera has to see, so this is a really difficult problem for a large amount of field. If it is like one strategical game in which the camera is moving in the direction of the player's view so it is like a single point of interest. And it becomes a more complicated problem when it is having a large field in which the camera moves freely around the entire field. Another role of a moderate player is a referee. In some game, required lots of efforts to detect complex decision with a large amount of accuracy. So, to implement this type of complex result the game would have need of pattern recognition method which can execute all the mediator activities [4].

\section{Open World Game \& Story Generation}

A game is a story that then progresses linearly, and might players have to choose some action from different range of actions for each stage [3]. For understanding properly let's consider some graphical states,

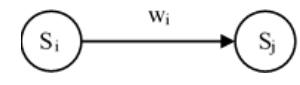

)

(b)

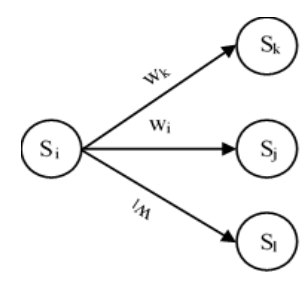

Fig. 2. Represent game states as in the form of a graph

In the above graph, we represent 2 different types of states method for any story games, for example in the above graph we see that $S_{i}$ represented as a present state, $S_{j}, S_{k}, S_{1}$ are next states, and $\mathrm{w}_{\mathrm{i}}, \mathrm{w}_{\mathrm{j}}, \mathrm{w}_{\mathrm{k}}, \mathrm{w}_{\mathrm{l}}$, the way to achieve that states. (a) in this case, this story moves in linear fashion means there is no other choice to move on and in

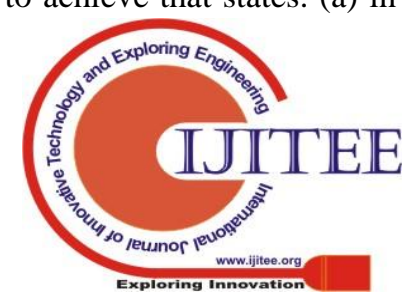


(b) case story moves further with having more options so, this type of open-world game moves with more no. of choices so this type of games provide freedom to the players during playing open-world story games. For example, Farcry, GTA Series, chess, etc. In the present world, every game is moving towards a realistic effect and for that, every game gives a realistic touch to the games [1].

The main purpose of adding a story into games in which player have freedom to choose between fixed entry points, so by choosing any entry points how the game story is going on means whether the story gets boring or having more amount of action plays so giving some surprising thing or provide some rest to the player, so for this kind of thing pattern recognition required.

\section{CONCLUSION}

In this, we define the games are a suitable application for pattern recognition. It is useful in the complete AI system by generating high-level results for any decision-making gaming world. Also, this pattern recognition method is useful for getting all the required details from the gaming world. In this, we discuss how the recognition method in games can be observed based on several points of view.

\section{ACKNOWLEDGMENT}

The authors wish to thank Head of Department, Principal, and Dean of Faculty of Technology and Engineering, Charotar University of Science and Technology, Changa for their suggestions, encouragement, guidance and support in undertaking the present work. Special thanks to the Management for their moral support and continuous encouragement.

\section{REFERENCES}

1. Kaukoranta, Timo \& Smed, Jouni \& Hakonen, Harri. (2003). "Role of Pattern Recognition in Computer Games."

2. M. Capps, P. McDowell, and M. Zyda. A future for entertainment-defense research collaboration. IEEE Computer Graphics and Applications.

3. G. Costikyan. I have no words \& I must design: Toward a critical vocabulary for games. In F. Mayr “ an (editor), “ Computer Games and Digital Cultures Conference Proceedings, pp. 9-33, Tampere, Finland.

4. Federation Internationale de Football Association. Laws of the game. Web page, Nov. 2002. http://www.fifa.com/refs/laws E.html

5. J.E. Laird and M. van Lent. Human-level AI's killer application: interactive computer games. AI Magazine.

6. RoboCup. Web page, Nov. 2002. http://www.robocup.org/.

7. R. Schalkoff. Pattern Recognition: Statistical, Structural and Neural .u.Approaches. John Wiley \& Sons, ONew York, NY, 1992.

8. S. Rabin, editor. AI Game Programming Wisdom. Charles River Media, Hingham, MA, 2002.

9. International Game Developers Association. Web page, Nov. 2002 http://www.igda.org/

10. Marill and Green, 1960 Marill T., Green D.M.Statistical recognition functions and the design of pattern recognizers IRE Trans. E (Mariusz Szwoch, 2015)electron. Computers, EC-9 (1960), pp. 472-477

11. Mariusz Szwoch, Wioleta Szwoch, "Emotion Recognition for Affect Aware Video Games", in Image Processing \& Communications Challenges 6, 2015, Volume 313, (ISBN: 978-3-319-10661-8)

12. Ugur Demir, Esam Ghaleb, Hazım Kemal Ekenel, "A Face Recognition Based Multiplayer Mobile Game Application", in Artificial Intelligence Applications and Innovations, 2014, Volume 436, (ISBN: 978-3-662-44653-9)

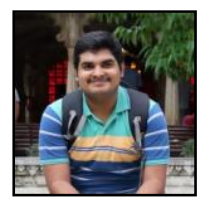

\section{AUTHORS PROFILE}

Dhruvil Parmar is pursuing B. Tech $\left[6^{\text {th }}\right.$ Semester $]$ in U \& P U. Patel Department of Computer Engineering at Chandubhai S. Patel Institute of Technology, CHARUSAT. His area of interest are Machine learning and Game development.

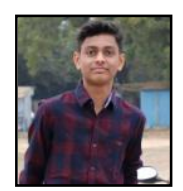

Mit Suthar is pursuing B. Tech $\left[6^{\text {th }}\right.$ Semester $]$ in U \& P U. Patel Department of Computer Engineering at Chandubhai S. Patel Institute of Technology, CHARUSAT. His area of interest are Machine learning and Game development.

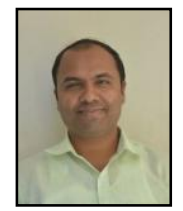

Dhaval Bhoi, working as an Assistant Professor in U \& P U. Patel Department of Computer Engineering and has more than 16 years of teaching experience in academics. $\mathrm{He}$ was awarded BE \& ME from DDU, Nadiad and currently pursuing his $\mathrm{Ph} . \mathrm{D}$. degree in the field of Data Mining. He has published more than 12 research papers in national and international journals and conferences. 